\title{
Parameter Characterization of High Latitude Geomagnetic Storms in 2010
}

\author{
Ye Su \\ Nanjing University of Information Science \& Technology, Nanjing, China \\ Email: Sue_1259@163.com
}

How to cite this paper: $\mathrm{Su}, \mathrm{Y}$. (2019). Parameter Characterization of High Latitude Geomagnetic Storms in 2010. Journal of Geoscience and Environment Protection, 7, 163-170. https://doi.org/10.4236/gep.2019.712011

Received: July 26, 2019

Accepted: December 20, 2019

Published: December 23, 2019

Copyright $\odot 2019$ by author(s) and Scientific Research Publishing Inc. This work is licensed under the Creative Commons Attribution International License (CC BY 4.0).

http://creativecommons.org/licenses/by/4.0/

(c) (i) Open Access

\begin{abstract}
Geomagnetic storm is a kind of severe disturbance that lasts for more than ten hours to several tens of hours in the entire Earth's magnetosphere. This paper uses the NRLMSISE-00 model to calculate the temperature and density data. According to the surveyed medium magnetic storm events, the characteristics of daily average density and temperature in the occurrence of magnetic storms in 2010 were studied. At the same time, high latitude meridians were taken as research objects. Divide the temperature and density characteristics at different heights. Results showed that the annual trend of density is the same for different heights, and there is an average of the average density every day on the six medium magnetic storm mountains. For the average daily temperature, each medium magnetic storm event corresponds to a temperature peak. The peak temperature on April 5 was the highest temperature throughout the year, consistent with the annual average density distribution. Due to the intensity of the magnetic storm, the temperature of a large area of bright areas rose sharply on April 12, which is also the highest in the year. At 18:00 on May 2, it was the peak time of the moderate magnetic storm event caused by CIR, but May 3 was the date of the peak temperature. This peak delay is reasonable.
\end{abstract}

\section{Keywords}

Magnetic Storm, High Latitude, Temperature, Density

\section{Introduction}

Geomagnetic storm is a kind of severe disturbance that lasts for more than ten hours to several tens of hours in the entire Earth's magnetosphere. It is one of the main disturbances of the Earth's magnetosphere. Gonzalez et al. (1994) argues that some geomagnetic storms, especially the extraordinarily large geo- 
magnetic storms, began with a sudden blast, marking the arrival of the interplanetary impact structure. This is usually consistent with the beginning of the increased stamping pressure (primary phase), followed by the continued southward interplanetary field (primary phase) and then back to normal (recovery phase).

Yi et al. (2018) found that the density of the middle layer in the Aurora region decreased significantly during the geomagnetic storm. The response of the intermediate layer density is immediate, exhibiting a measurable response in less than one day of the occurrence of a geomagnetic storm (the optimal resolution of our meteor radar density).

Li et al. (2018) used TIMEGCM to simulate the effect of geomagnetic storm on MLT temperature in the middle and low latitudes. Yuan et al. (2019) found that during the daytime, the enhanced density of the thermal layer on the GRACE orbit was slower than at night, as the EUV-driven polar wind suppressed the equator. At the same time, due to the main thermal expansion in the low latitude area, it is easy to pass through the equator area during daytime density enhancement.

Deng et al. (2018) used non-static GITM simulations to study the possible effects of extreme storms on the upper atmosphere. Joshua et al. (2014) has studied the response of the ionospheric F2 layer peak parameters to magnetic storms.

During the geomagnetic storms from August 3rd to 4th, 2010 and August 5th to 6th, 2011, the density and ion temperature were measured on the American continent using DMSP-F15 and F17 satellites to study (Valladares et al., 2017). The formation of a dynamic increase in plasma density in these processes. Kumar \& Parkinson (2017) found that high-latitude plasma density increased by 60 hours at medium intensity before the midnight equinox and the actual summer magnetic storm.

Shi et al. (2014) found that there were 8 magnetic storm events in 2010, which were February 15, April 6, April 12, May 2, May 29, August 4, October 11 and December 28th. Among them, the $70^{\circ} \mathrm{N}$ latitude in the high latitude and the 6 medium magnetic storm events in May were selected as the main research objects. And on May 3 (one day after the storm) and May 15 (when there is no storm) as a comparison object.

\section{Material and Methodology}

The data used in this article comes from the NRLMSISE-00 model web page calculation, which is mainly the temperature and density of the whole year of 2010. The data is averaged over the circle and then divided into heights. Then, the date data of the occurrence of the magnetic storm is selected and compared with the date of the storm less date. Figure 1 is the NRLMSIS-00 model calculation interface ( https://ccmc.gsfc.nasa.gov/modelweb/models/nrlmsise00.php).

Picone et al. (2002) describes the NRLMSISE-00 empirical atmospheric model and its scientific use. The NRLMSISE-00 empirical atmospheric model extends 


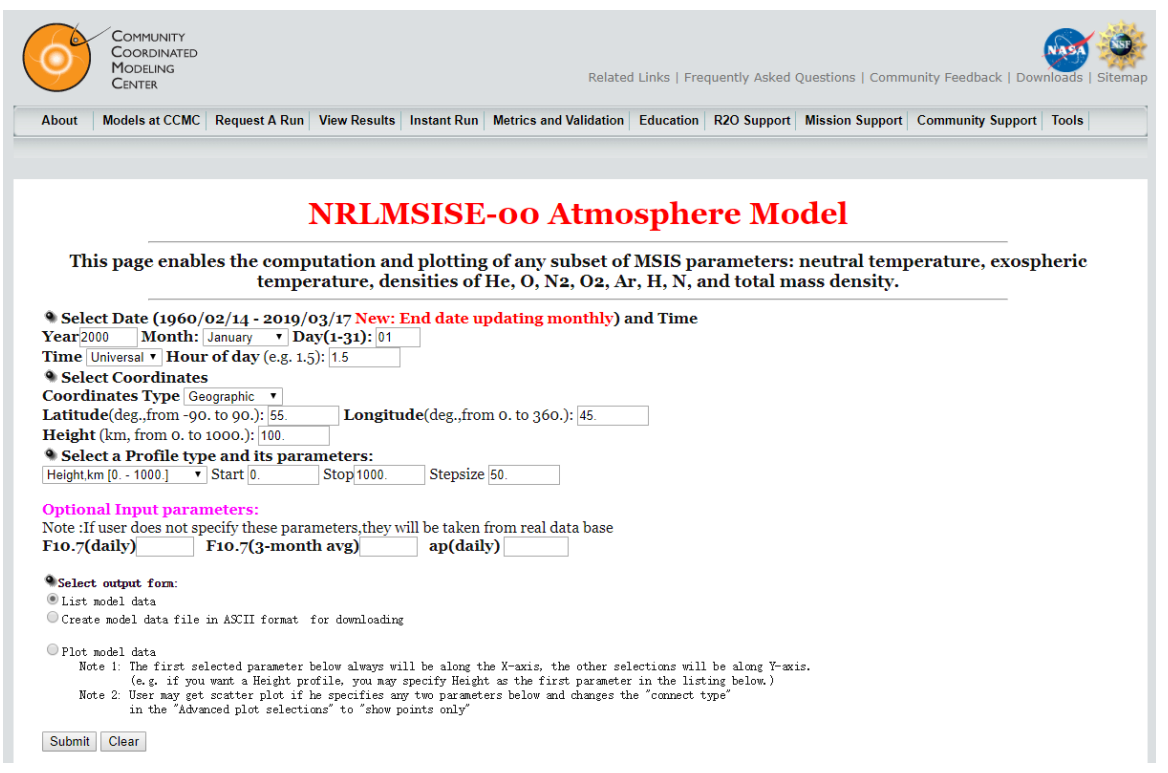

Figure 1. The NRLMSIS-00 model calculation interface.

from the ground to the exobase, which is a major upgrade of the MSISE-90 model in the thermal layer. Shi et al. (2015) believes that at the heights of 500 and $600 \mathrm{~km}$, as the accuracy of the NRLMSISE00 model decreases, the calibration scale changes more. This may affect the mapping error and our characterization of the storm time parameters.

\section{Results and Discussion}

\subsection{Annual Variation of the Average Density of $70^{\circ} \mathrm{N}$ in 2010}

Figure 2 describe the annual average density distribution at three heights of 200 $\mathrm{km}, 400 \mathrm{~km}$ and $600 \mathrm{~km}$. Since the upper and lower limits of the daily average density of each height are too large, it is shown on the same graph that the variation will be reduced, which is not conducive to the analysis of the graph. The dotted line marks the date of the occurrence of six moderate magnetic storm events, which are April 5, April 12, May 2, May 29, August 4, and October 11. The horizontal axis is the date and the vertical axis is the density. Through the observation of the three images, we can see that the annual trend of the height density is the same, and there is a daily average density peak on the mountain of the six medium magnetic storms. The peak value of the density on April 5 is the maximum for the whole year. In the internal relationship between density and the magnitude of the magnetic storm, we can infer that in the six medium magnetic storms, the intensity of the magnetic storm on April 5 should be slightly larger than the other five. Therefore, the fluctuations in atmospheric density are greater.

\subsection{Annual Average Temperature of $70^{\circ} \mathrm{N}$ in 2010}

The annual average temperature distribution map is the same as the annual average density annual distribution map at height of $200 \mathrm{~km}, 400 \mathrm{~km}$ and $600 \mathrm{~km}$ 

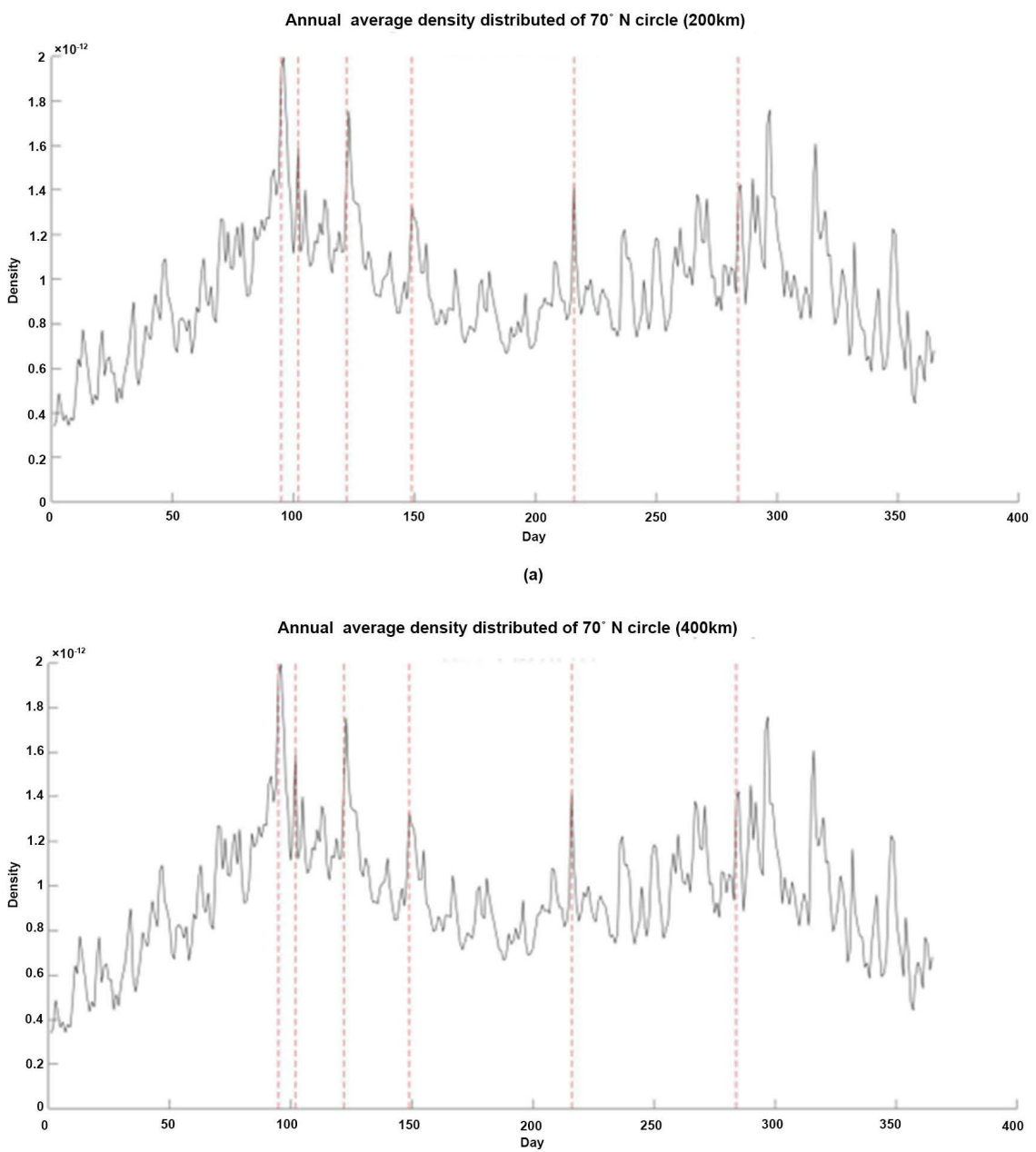

(b)

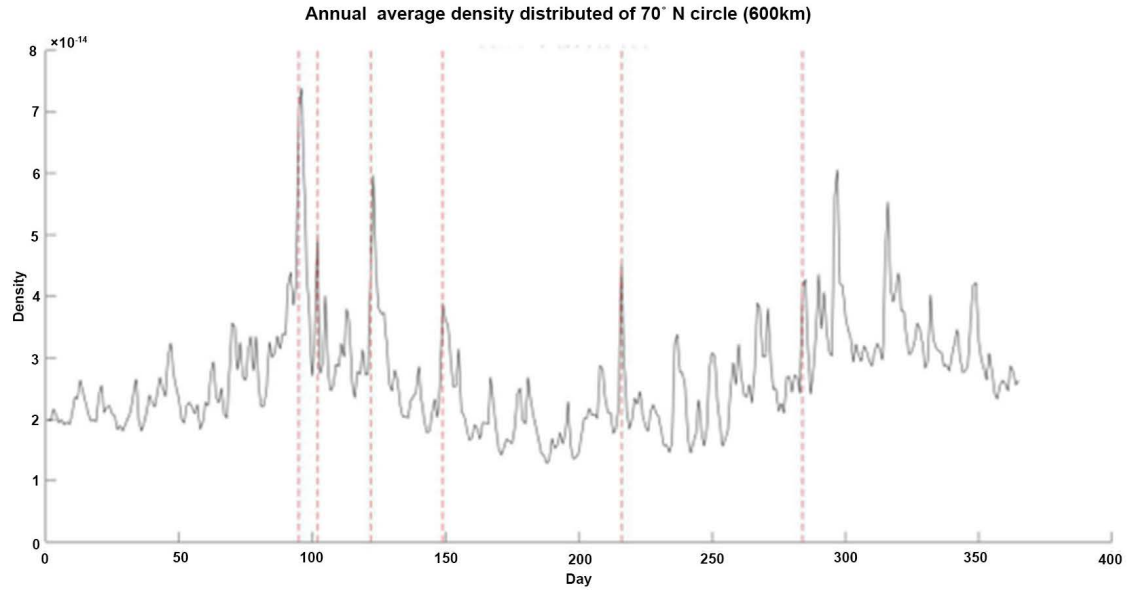

(c)

Figure 2. Average daily density of $70^{\circ} \mathrm{N}$ at a height of (a) $200 \mathrm{~km}$, (b) $400 \mathrm{~km}$ and (c) 600 $\mathrm{km}$.

(Figure 3). It is only because the temperature is the same in the upper and lower limits of the three heights in the previous year, so it can be drawn on a graph for analysis. The blue line indicates the annual average temperature distribution 


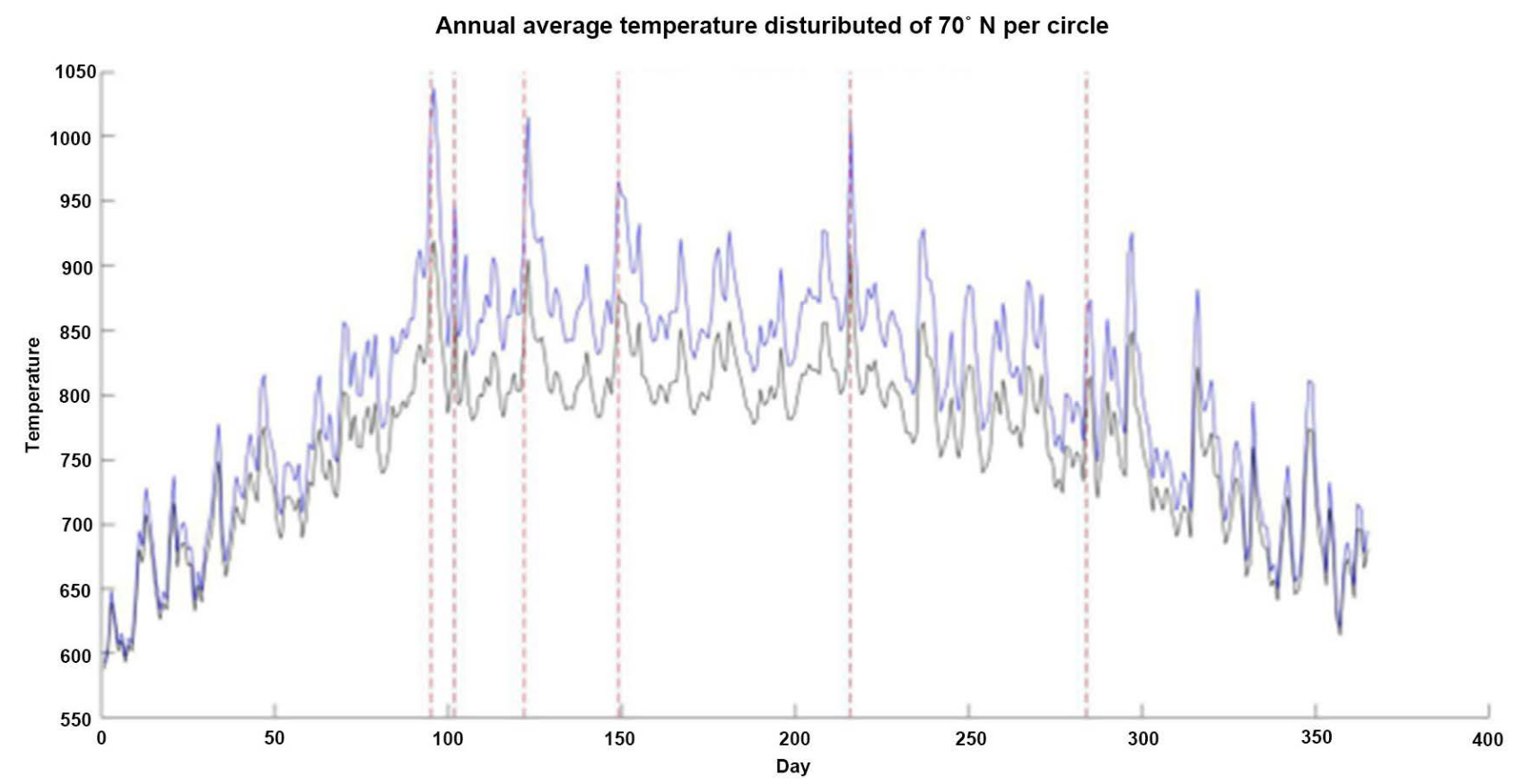

Figure 3. Average daily temperature of $70^{\circ} \mathrm{N}$ at a height of $200 \mathrm{~km}, 400 \mathrm{~km}$ and $600 \mathrm{~km}$.

map of $400 \mathrm{~km}$ and $600 \mathrm{~km}$ (the two height curves coincide), and the black line indicates the annual average temperature distribution map of $200 \mathrm{~km}$. The dotted line indicates the date of six medium magnetic storm events. As can be seen from the figure, each medium magnetic storm event corresponds to a temperature peak, and the temperature peak corresponding to April 5 is the maximum temperature of the whole year. This result is consistent with the annual average density annual distribution.

\subsection{Changes in the Average Daily Temperature at $70^{\circ} \mathrm{N}$ in March, April and May 2010}

Figure 4 shows the daily average temperature distribution from $200 \mathrm{~km}$ to 800 $\mathrm{km}$, and the right color bar shows the gradual increase in temperature from dark to bright. Among them, the March region without moderate magnetic storms is significantly darker than the April and May with mid-level magnetic storms. Among them, the large area of bright areas on April 12 showed a sharp increase in temperature due to the intensity of the magnetic storm, which is also the maximum for the whole year. There is also a very bright area on May 2nd. The magnetic storm event on May 29 and April 12 has no obvious bright areas corresponding to the time zone. The image is basically the same as previously analyzed.

\subsection{Changes in the Average Daily Temperature in May 2010 at $70^{\circ} \mathrm{N}$}

Figure 5 shows the daily average temperature as a function of height in May. The red dotted line indicates May 2, the green dotted line indicates May 3, and 
$70^{\circ} \mathrm{N}$ daily average distributed with height (March, April, May)

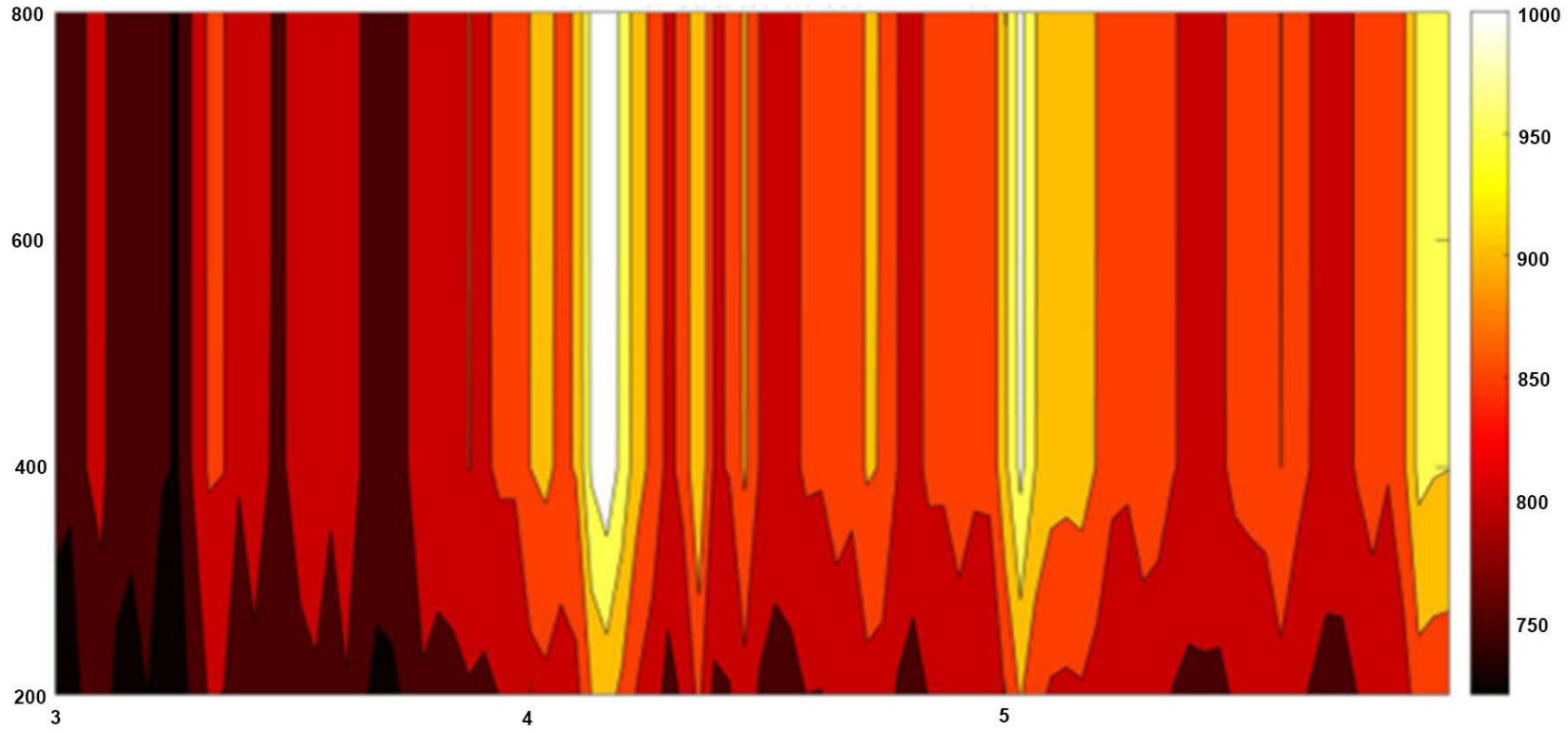

Figure 4. Daily average temperature distribution of $70^{\circ} \mathrm{N}$ with height in March, April and May (The number 3, 4 and 5 denote March, April and May, respectively).

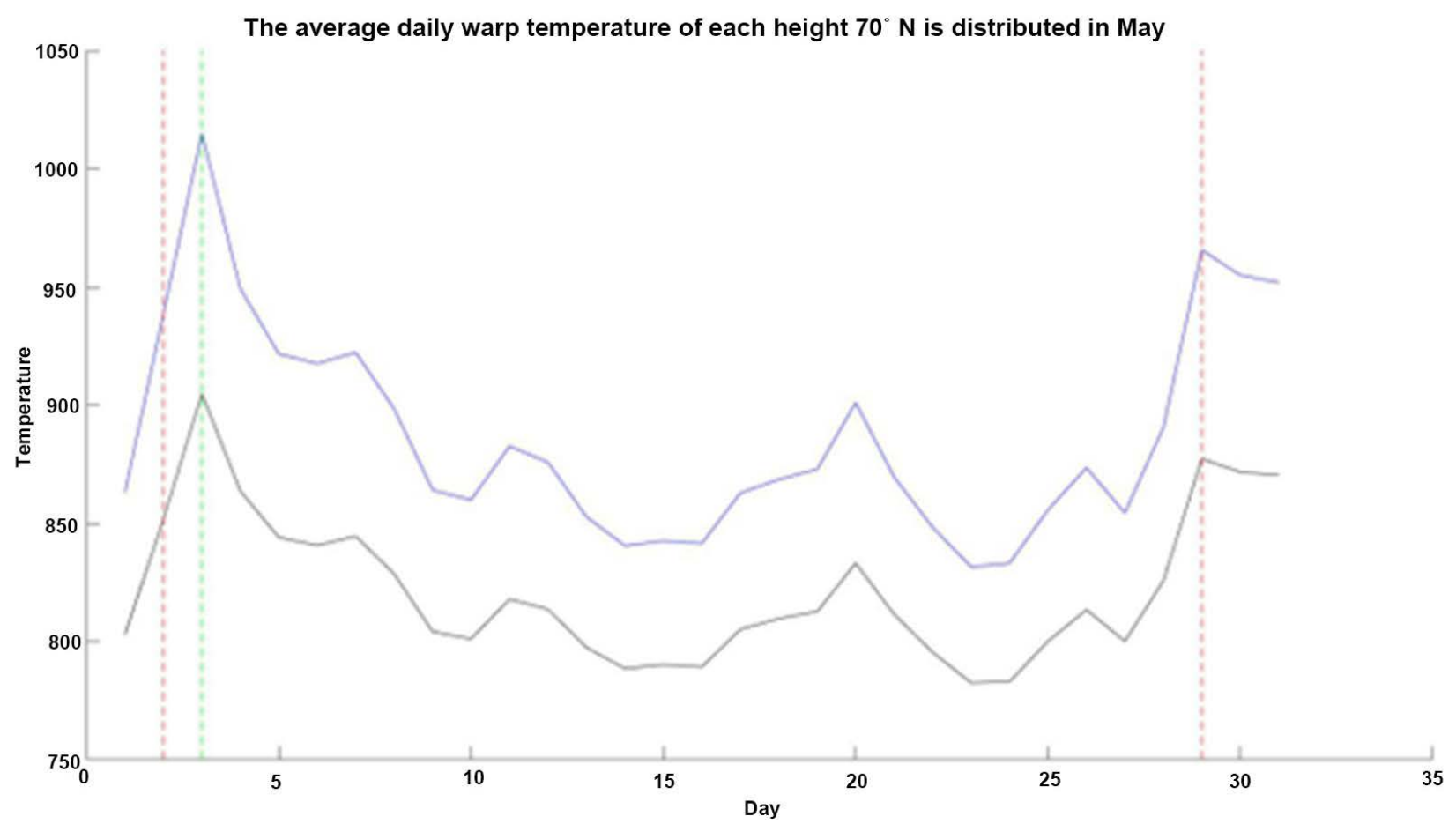

Figure 5. Daily average temperature distribution of $70^{\circ} \mathrm{N}$ with height in May.

the brown dotted line indicates May 29. Shi et al. (2014) The Dst index study found that 18:00 on May 2 was the peak time for a moderate magnetic storm event caused by CIR (co-transition interaction zone). However, as can be seen from the figure, May 3 is the date of the peak temperature. In the annual distribution map and the March, April, and May distribution maps, due to the small 
date interval, the date corresponding to the specific peak cannot be obtained, but drawing separately in May, each date interval is larger, and the time corresponding to the peak temperature can be seen more clearly. The peak intensity time of the magnetic storm is different from the peak temperature time. The Yeeram (2017) study that the storm time (primary and main phase) associated with CIR is more than one day. In other words, the time depends on the duration of the magnetic reconnection during the main phase of the storm. Under the same principle, Qiu (2015) found that the time of occurrence of ionospheric storms is mostly between -6 and $6 \mathrm{~h}$, but the range of ionospheric storms caused by CIR is wider, between -12 and $24 \mathrm{~h}$. The ionospheric burst caused by CME is mainly between -6 and 6 hours. Therefore, the peak delay phenomenon presented in Figure 5 is reasonable.

\section{Conclusion}

For different heights, the annual trend of density is the same, and there is an average of the average density every day on the six middle magnetic storm mountains. The density increase on April 5 of the year of 2010 can reach the highest value. The magnetic storm intensity on this day should be slightly greater than the other six. Therefore, fluctuations in atmospheric density are greater.

For daily average temperatures at altitudes of $200 \mathrm{~km}, 400 \mathrm{~km}$, and $600 \mathrm{~km}$, each magnetic storm event corresponds to a temperature peak, and the temperature peak corresponding to April 5 is the highest temperature throughout the year. This result is consistent with the annual average annual density distribution.

Due to the intensity of the magnetic storm, the temperature of a large area of bright areas rose sharply on April 12, which is also the highest in the year. There was also a very bright area on May 2. The magnetic storm events of May 29 and April 12 did not correspond to significant bright areas at that time.

At 18 o'clock on May 2 is the peak time of moderate magnetic storm events caused by CIR. However, it can be seen from the figure that May 3 is the date of the peak temperature. This peak delay is reasonable.

\section{Conflicts of Interest}

The author declares no conflicts of interest regarding the publication of this paper.

\section{References}

Deng, Y., Sheng, C., Tsurutani, B. T., \& Mannucci, A. J. (2018). Possible Influence of Extreme Magnetic Storms on the Thermosphere in the High Latitudes. Space Weather, 16, 802-813. https://doi.org/10.1029/2018SW001847

Gonzalez, W. D., Joselyn, J. A., Kamide, Y., Kroehl, H. W., Rostoker, G., Tsurutani, B. T. et al. (1994). What Is a Geomagnetic Storm? Journal of Geophysical Research, 99, 5771. https://doi.org/10.1029/93JA02867

Joshua, B. W., Adeniyi, J. O., Adimula, I. A., Oladipo, O. A., Olawepo, O. A., \& Adebiyi, 
S. J. (2014). Ionospheric Response to the Storm-Time Disturbance of 29 May, 2010. Advances in Space Research, 53, 219-225. https://doi.org/10.1016/j.asr.2013.11.003

Kumar, V. V., \& Parkinson, M. L. (2017). A Global Scale Picture of Ionospheric Peak Electron Density Changes during Geomagnetic Storms. Space Weather, 15, 637-652. https://doi.org/10.1002/2016SW001573

Li, J., Wang, W., Lu, J., Yuan, T., Yue, J., Liu, X. et al. (2018). On the Responses of Mesosphere and Lower Thermosphere Temperatures to Geomagnetic Storms at Low and Middle Latitudes. Geophysical Research Letters.

Picone, J. M., Hedin, A. E., Drob, D. P., \& Aikin, A. C. (2002). NRLMSISE-00 Empirical Model of the Atmosphere: Statistical Comparisons and Scientific Issues. Journal of Geophysical Research: Space Physics, 107, SIA 15-1-SIA 15-16.

https://doi.org/10.1029/2002JA009430

Shi, C., Li, W., Li, M., Zhao, Q., \& Sang, J. (2015). Calibrating the Scale of the NRLMSISE00 Model during Solar Maximum Using the Two Line Elements Dataset. Advances in Space Research, 56, 1-9. https://doi.org/10.1016/j.asr.2015.03.024

Shi, L., Shen, C., \& Wang, Y. (2014). The Interplanetary Origins of Geomagnetic Storm with Dst(min) $\leq-50 \mathrm{nT}$ in 2007-2012. Chinese Journal of Geophysics, 57, 3822-3833.

Valladares, C. E., Eccles, J. V., Basu, S., Schunk, R. W., Sheehan, R., Pradipta, R. et al. (2017). The Magnetic Storms of 3-4 August 2010 and 5-6 August 2011: 1. Ground- and Space-Based Observations. Journal of Geophysical Research: Space Physics. https://doi.org/10.1002/2016JA023359

Yeeram, T. (2017). Interplanetary Drivers of Daytime Penetration Electric Field into Equatorial Ionosphere during CIR-Induced Geomagnetic Storms. Journal of Atmos pheric and Solar-Terrestrial Physics, 157-158, 6-15. https://doi.org/10.1016/j.jastp.2017.02.008

Yi, W., Reid, I. M., Xue, X., Murphy, D. J., Hall, C. M., Tsutsumi, M. et al. (2018). Highand Middle-Latitude Neutral Mesospheric Density Response to Geomagnetic Storms. Geophysical Research Letters, 45, 436-444. https://doi.org/10.1002/2017GL076282

Yuan, L., Jin, S., \& Calabia, A. (2019). Distinct Thermospheric Mass Density Variations Following the September 2017 Geomagnetic Storm from GRACE and Swarm. Journal of Atmospheric and Solar-Terrestrial Physics, 184, 30-36.

https://doi.org/10.1016/j.jastp.2019.01.007 